\title{
Anti-TNF antibodies do not induce the apoptosis of lamina propria mononuclear cells in uninflamed intestinal tissue in patients with Crohn's disease
}

\author{
Piotr Eder ${ }^{1}$, Liliana Lykowska-Szuber ${ }^{1}$, Iwona Krela-Kazmierczak ${ }^{1}$, \\ Kamila Stawczyk-Eder ${ }^{1}$, Karolina Sterzynska², Katarzyna Iwanik ${ }^{3}$, \\ Przemyslaw Majewski ${ }^{3}$, Maciej Zabel², Krzysztof Linke ${ }^{1}$
}

${ }^{1}$ Department of Gastroenterology, Human Nutrition and Internal Diseases, Poznan University of Medical Sciences

${ }^{2}$ Department of Histology and Embryology, Poznan University of Medical Sciences

${ }^{3}$ Department of Clinical Pathomorphology, Poznan University of Medical Sciences

\begin{abstract}
It is not known if anti-tumor necrosis factor (anti-TNF) agents provoke only apoptosis of lamina propria mononuclear cells (LPMC) engaged in inflammatory processes or whether it's a general phenomenon concerning all LPMC. In this study we carried out an immunohistochemical analysis of the expression of several apoptosis-related proteins (active caspase-3, Bax, Bcl-2, Fas, TNFR1, CD4, and CD8) in uninflamed mucosa in Crohn's disease (CD) patients treated with anti-TNF agents. $16 \mathrm{CD}$ patients (mean age $34 \pm 11$, mean disease duration $7 \pm 5$ years) were included in the study. 10 patients were treated with infliximab and 6 - with adalimumab. The expression of active caspase 3, Bax, Bcl-2, Fas, TNFR1 and CD8 in LPMC did not change significantly after the therapy. We concluded that anti-TNF antibodies did not promote LPMC apoptosis in uninflamed tissues. This is in contrast to the phenomena observed in inflamed tissues. These data show that anti-TNF antibodies rather restore the susceptibility to apoptosis of LPMC in inflamed areas of the gut in CD, than directly induce LPMC apoptosis; otherwise the anti-TNF antibodies should have also induced apoptosis in the uninflamed mucosa. (Folia Histochemica et Cytobiologica 2013, Vol. 51, No. 3, 239-243)
\end{abstract}

Key words: apoptosis; Crohn's disease; infliximab; adalimumab; mononuclear cells; uninflamed mucosa

\section{Introduction}

The mechanisms of action of anti-TNF antibodies (infliximab, IFX, and adalimumab, ADA) used in the treatment of Crohn's disease (CD) are still not well known. It is believed that one of the crucial phenomena leading to the down-regulation of inflammatory infiltration in the intestinal tissue is the induction of apoptosis of immune cells [1-3]. Study from our group showed that the proportion of apoptotic lamina

Correspondence address: P. Eder, Chair and Department of Gastroenterology, Human Nutrition and Internal Diseases, University of Medical Sciences, Przybyszewskiego St. 49, 60-355 Poznan, Poland; tel.: +48 6186913 43; fax: +48 6186916 86; e-mail: piotr.eder@op.pl propria mononuclear cells (LPMC) is increased after anti-TNF therapy, as evidenced in mucosal biopsies taken from inflamed areas of the colon in patients with CD [4]. However, the question remains whether anti-TNF antibodies also induce programmed cell death of LPMC in uninflamed areas of the gut.

In the current study we carried out an immunohistochemical analysis of the effect of IFX and ADA on the expression of apoptosis-related proteins in LPMC in intestinal biopsies taken from uninflamed areas of the colon mucosa in patients with $\mathrm{CD}$.

\section{Material and methods}

The inclusion criterion for the study was an exacerbation of the disease in spite of the administration of 
maximal doses of standard therapy for $\mathrm{CD}(3-4 \mathrm{~g}$ daily of aminosalicylates for at least 12 weeks, steroids in stable doses for at least 4 weeks and/or azathioprine $2-2.5 \mathrm{mg} / \mathrm{kg}$ body weight for at least 12 weeks) [5]. 16 patients participated in the study ( 9 females and 7 males, mean age $34 \pm 11$ years). Mean CD duration was $7 \pm 5$ years.

The study was approved by the Bioethics Committee of the Poznan University of Medical Sciences. After a written consent was obtained, each patient underwent a colonoscopy before starting anti-TNF therapy. Multiple biopsies were taken from the uninflamed (macroscopically normal) areas of the colon. Disease clinical activity was assessed by calculating the Crohn's Disease Activity Index (CDAI). The patients then received either three doses of IFX $5 \mathrm{mg} / \mathrm{kg}$ body weight intravenously in weeks 0,2 and 6 , or ADA $160 \mathrm{mg}$ subcutaneously in week $0,80 \mathrm{mg}$ in week 2 , and $40 \mathrm{mg}$ every other week until week 12 , in accordance with the current recommendations with regard to biological therapy [5]. 10 patients were treated with IFX and 6 with ADA. After the induction of anti-TNF therapy was finished, the patients were admitted to our Department to carry out follow-up investigations. These also included colonoscopy with tissue sampling from the same uninflamed areas of the colon.

Tissue samples obtained during colonoscopy were fixed in $10 \%$ neutral buffered formalin and then embedded in paraffin. $\mathrm{H}+\mathrm{E}$ staining was performed and immunohistochemical expression (the $\mathrm{ABC}$ technique, in accordance with Hsu et al.) of selected proteins was assessed in LPMC as previously described $[4,6]$. The following primary antibodies were used: anti-active caspase 3 (R\&D Systems, Minneapolis, USA, No AF835, concentration $1 \mu \mathrm{g} / \mathrm{mL}$ ), anti Bcl-2 (DAKO, Glostrup, Denmark, No M088701, dilution 1:500), anti-Bax (DAKO, No A353301, dilution 1:2000), anti-Fas (Leica Monocastra, Newcastle, UK, No NCL-FAS-310, dilution 1:200), anti-TNFR1 (Abcam, San Diego, USA, No Ab 19139, dilution 1:2000), anti-CD4 (DAKO, No M731001, dilution 1:200) and anti-CD8 (DAKO, No M710301, dilution 1:300). Semiquantitative 12-point Remmele and Stegner Immunoreactive Score (IRS) was used to assess the expression of active caspase 3, Bcl-2 and Bax. IRS takes into account the percentage of positive cells $(0 \%-0$ pts; $<10 \%-1$ pt; $11-50 \%-2$ pts; $51-80 \%-3 \mathrm{pts} ;>80 \%-4 \mathrm{pts})$ and the intensity of staining (graded 0-3 pts) [7]. To obtain the final score of protein expression the aforementioned components of IRS were multiplied. In case of CD4, CD8, Fas, and TNFR1 a modified HercepTest was applied. This score takes into account the intensity and completeness of membrane staining, as well as the number of positive cells, and is graded 0-3 pts [8].

The appropriate statistical analysis was performed. The differences in proteins expression were evaluated using the Wilcoxon non-parametric test. Statistical significance was assumed at $\mathrm{p}<0.05$.

\section{Results and discussion}

The biological therapy resulted in a statistically significant decrease of CDAI (266 vs. 98 pts; $\mathrm{p}<0.05$ ).

$\mathrm{H}+\mathrm{E}$ staining did not reveal any histological signs of active inflammation in the lamina propria of the endoscopically-uninflamed intestinal tissue samples. We found the immunohistochemical expression of investigated proteins mainly in LPMC and enterocytes, single polymorphonuclear cells were also immunoreactive (Figure 1). The expression of an active caspase 3, Bax, Bcl-2 (as a well as a pro-apoptotic Bax/ /Bcl-2 ratio), Fas, TNFR1 and CD8 in LPMC did not change significantly after the anti-TNF therapy. Only the number of CD4 LPMC decreased significantly (Figure 2). The expression of the investigated proteins in the epithelial cells was also quantified. However, anti-TNF treatment did not change the expression of any of the assessed proteins in the enterocytes.

In our study we tried to answer the question whether anti-TNF agents provoke only the apoptosis of the LPMC engaged in inflammatory processes or whether it is a general phenomenon affecting all LPMC, also in uninflamed mucosa. This question is essential because it was shown that both ADA and IFX can induce apoptosis of peripheral blood monocytes, which are not directly involved in the inflammatory processes in the intestines in IBD $[9,10]$. On the other hand, Ten Hove et al. showed that only CD3/CD28-stimulated Jurkat T cells undergo apoptosis after the administration of IFX, as opposed to unstimulated Jurkat T cells [11].

The main finding of this study is the demonstration that LPMC from uninflamed areas of the gut did not die in programmed cell death mechanism after anti-TNF antibody treatment. This confirms that IFX and ADA regulate disturbed apoptosis of inflammatory cells sensitizing LPMC to pro-apoptotic stimuli rather than provoke a programmed cell death by itself. The susceptibility to apoptosis of LPMC is locally down-regulated probably due to the influence of several proinflammatory cytokines. For example IL-6, which is present at the site of inflammation in high concentration, in complex with its soluble receptor, can bind to membrane gp130 protein in lymphocytes $[12,13]$. This results in signal transducer and activator of transcription 3 (STAT3) activation. STAT3 induces the expression of genes encoding the 

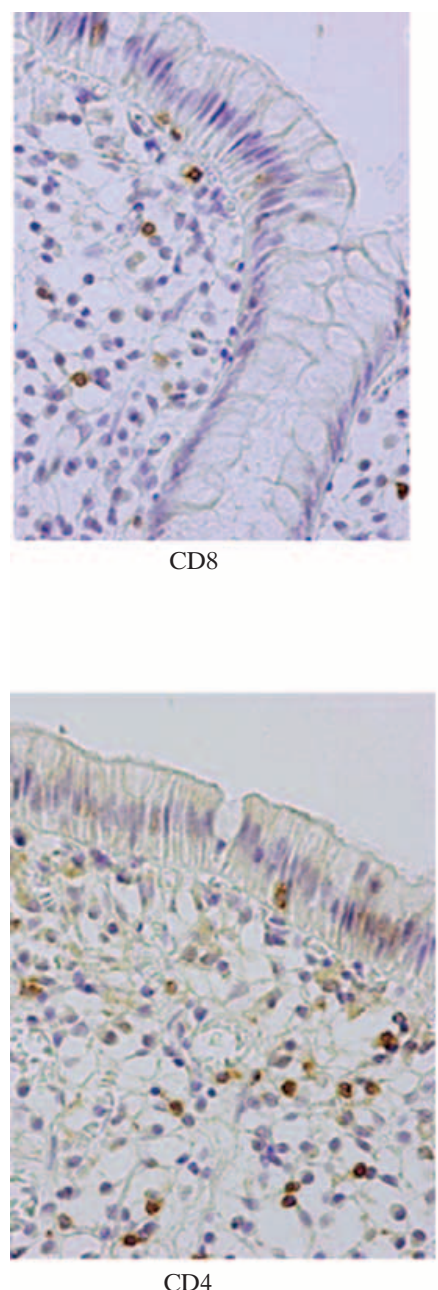

$\mathrm{CD} 4$

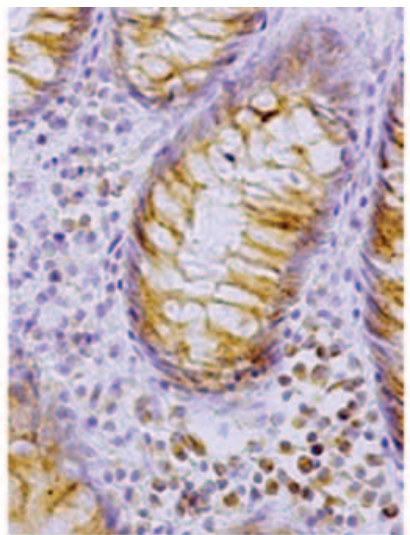

Bax

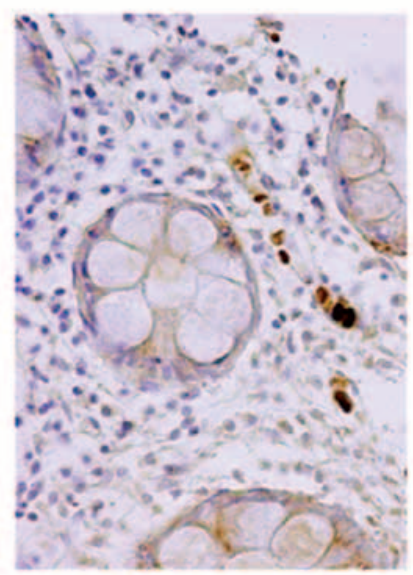

Active caspase 3

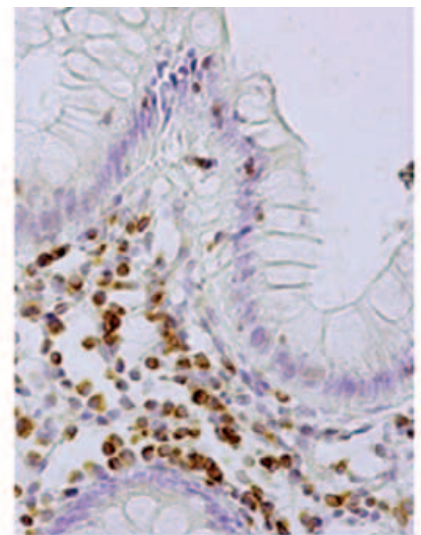

Bcl-2

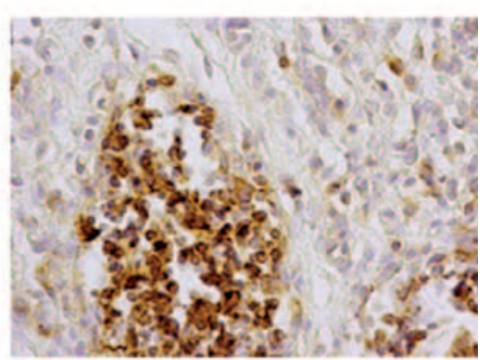

Fas

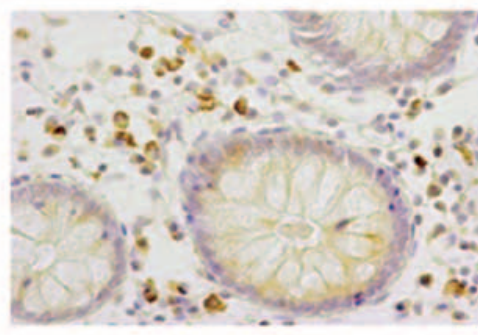

TNFR1

Figure 1. Immunohistochemical localization of active caspase 3, CD4, CD8, Bax, Bcl-2, Fas and TNFR1 in colonic biopsies taken from uninflamed mucosa of a $\mathrm{CD}$ patient. $\mathrm{ABC}$ technique. Objective magnification $\times 40$; nuclei were counterstained with hematoxylin
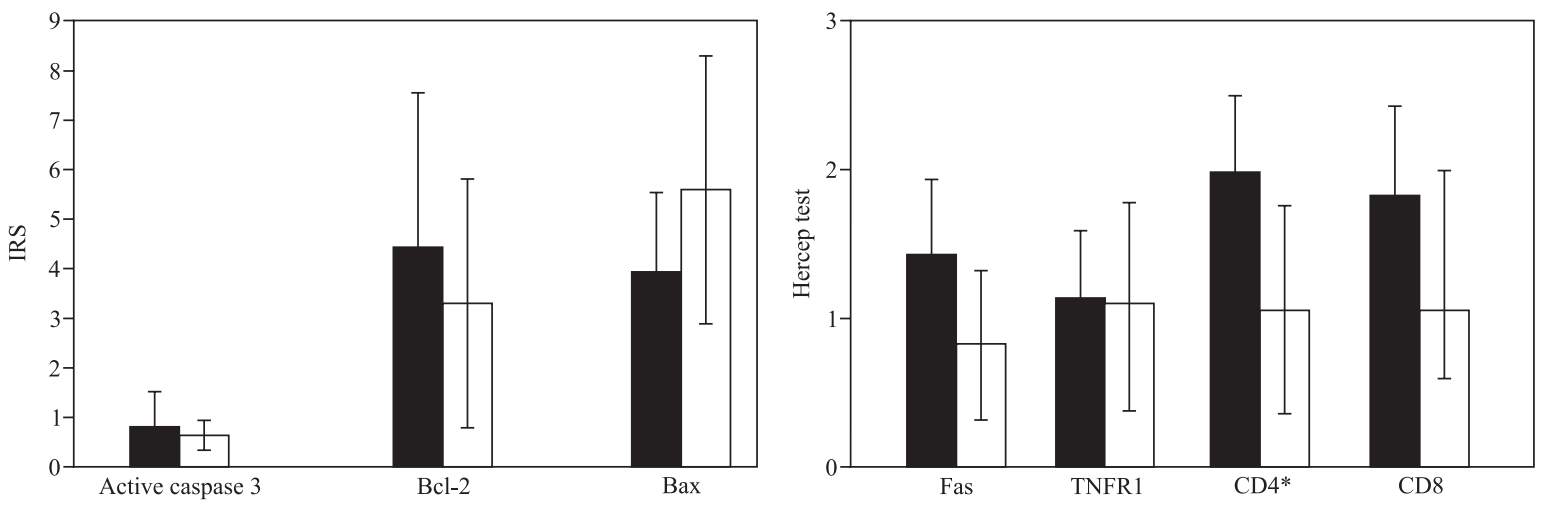

Figure 2. The influence of anti-TNF antibodies on the expression of (A): an active caspase 3 Bcl-2, Bax; (B): Fas, TNFR1, CD4 and CD8 in lamina propria mononuclear cells of uninflamed mucosa of CD patients. IRS - immunoreactive score. $* \mathrm{p}<0.05$ 
anti-apoptotic proteins belonging to Bcl-2 family proteins like Bcl-2 or BclX. Another cytokine which can be involved in this phenomenon is IL-12 [13]. IL-12 after binding to its receptor can activate the STAT4 pathway, thereby inhibiting the activation of initiator caspases. This interrupts the activation of caspase cascade leading to disturbances in apoptotic cellular elimination. IFX and ADA, being strong anti-inflammatory drugs, down-regulate the local concentration of IL-6 and IL-12, which, in turn, sensitizes LPMC to pro-apoptotic stimuli. This leads to the stimulation of inflammatory cells' programmed cell death and reduces the inflammatory infiltration in the gut. These phenomena do not affect the uninflamed tissue in CD, because here, the apoptosis of LPMC is probably not initially disturbed.

Another possible explanation of the difference in effect of IFX or ADA on LPMC apoptosis in inflamed and uninflamed tissues is the difference in cellular milieu. Atreya et al. showed that anti-TNF antibodies are able to induce T-cell apoptosis only when they are co-cultured with transmembrane TNF-expressing (tmTNF) CD14 macrophages [2]. The authors speculated that macrophages in inflamed mucosa stimulate lymphocytes' resistance to apoptosis by binding of their tmTNF to TNFR 2 on T cells. IFX and ADA bind to tmTNF on macrophages and in that way may interfere with the tmTNF/TNFR 2 pathway. Thus, anti-TNF antibodies indirectly provoke the programmed cell death of $\mathrm{T}$ cells.

In other studies it was shown that there is a decrease in the number of immune suppressor cells in inflamed mucosa in $\mathrm{CD}$ and/or their functions are altered. Moreover, anti-TNF therapy restores mucosal homeostasis and supports immunosuppressive reactions, which are disturbed under inflammatory conditions. A study by Ricciardelli et al. revealed that the number of mucosal CD4 CD25 (FOXP3+) $T$ regulatory cells was significantly reduced in active $\mathrm{CD}$, when compared with healthy conditions. This was reversed after IFX administration, but not after the conventional CD therapy [14]. Vos et al. showed also that anti-TNF antibodies induce regulatory CD206 macrophages in an Fc-dependent signal pathway [15]. This leads to the inhibition of activated and immunestimulated proliferation of T cells. In summary, it can be hypothesized that the restoration of the suppressive functions of different cell types (which function is altered in inflamed mucosa) after anti-TNF antibodies administration, can indirectly enhance the apoptotic elimination of mucosal T cells.

Another observation in our study is the significant decrease in the number of CD4 LPMC in healthy tissues. CD4 cells are believed to be the most important inflammatory cells engaged in CD pathogenesis. Anti-TNF antibodies can probably induce the apoptosis of peripheral blood CD4 cells and down-regulate the CD4 migration from the blood vessel into the tissues [9]. One can speculate that those two aforementioned phenomena can explain the significant decrease in CD4 LPMC also in the uninflamed intestinal tissue after the administration of anti-TNF agents.

\section{Conclusions}

We have for the first time, to the best of our knowledge, directly demonstrated in intestinal specimens, that anti-TNF antibodies do not promote LPMC apoptosis in the uninflamed colon mucosa in CD. This suggests that the induction of LPMC apoptosis in inflamed areas of the intestines observed after the administration of anti-TNF agents may represent a secondary phenomenon to the down-regulation of proinflammatory and anti-apoptotic cytokine concentration, or secondary to the restoration of the altered cellular milieu.

\section{Acknowledgements}

The study was supported by the grant from the Polish Ministry of Science and Higher Education (NN401073337).

\section{References}

1. Lugering A, Lebiedz $\mathrm{P}$, Koch $\mathrm{S}$ et al. Apoptosis as a therapeutic tool in IBD? Ann NY Acad Sci. 2006;1072:62-77.

2. Atreya R, Zimmer M, Bartsch B et al. Antibodies against tumor necrosis factor (TNF) induce T-cell apoptosis in patients with inflammatory bowel diseases via TNF Receptor 2 and intestinal CD14+ macrophages. Gastroenterology. 2011;141:2026-2038.

3. Caprioli F, Bose F, Rossi L et al. Reduction of CD68+ macrophages and decreased IL-17 expression in intestinal mucosa of patients with inflammatory bowel disease strongly correlate with endoscopic response and mucosal healing following infliximab therapy. Inflamm Bowel Dis. 2013;4:729-739.

4. Eder P, Lykowska-Szuber L, Krela-Kazmierczak I et al. The influence of infliximab and adalimumab on the expression of apoptosis-related proteins in lamina propria mononuclear cells and enterocytes in Crohn's disease - An immunohistochemical study. J Crohns Colitis. 2013;7:706-716.

5. Dignass A, Van Assche G, Lindsay JO et al. The second European evidence-based consensus on the diagnosis and management of Crohn's disease: current management. J Crohns Colitis. 2010;4:28-62.

6. Hsu SM, Raine L, Fanger H. Use of avidin-biotin peroxidase complex (ABC) in immunoperoxidase techniques: a comparison between ABC and unlabeled antibody (PAP) procedures. J Histochem Cytochem. 1981;29: $577-580$. 
7. Remmele W, Stegner HE. Vorschlag zur einheitlichen Definition eines immunreaktiven Score (IRS) fur den Immunohistochemichen Ostrogenrezeptor-Nachweis (ER-ICA) im Mammikarzinomgewebe. Patologie. 1987;8:138-140.

8. Ruschoff J, Dietel M, Baretton G et al. HER2 diagnostics in gastric cancer-guideline validation and development of standardized immunohistochemical testing. Virchows Arch. 2010;457:299-307.

9. Tracey D, Klareskog L, Sasso EH et al. Tumor necrosis factor antagonist mechanisms of action: a comprehensive review. Pharmacol Ther. 2008;117:244-279.

10. Shen C, Assche GV, Colpaert S et al. Adalimumab induces apoptosis of human monocytes: a comparative study with infliximab and etanercept. Aliment Pharmacol Ther. 2005;21:251-258.
11. Ten Hove T, van Montfrans C, Peppelenbosch MP et al. Infliximab treatment induces apoptosis of lamina propria $\mathrm{T}$ lymphocytes in Crohn's disease. Gut. 2002;50:206-211.

12. Ito H. IL-6 and Crohn's disease. Curr Drug Targets Inflamm Allergy. 2003;2:125-130.

13. Mudter J, Neurath MF. Apoptosis of T cells and the control of inflammatory bowel disease: therapeutic implications. Gut. 2007;56:293-303.

14. Ricciardelli I, Lindley KJ, Londei M et al. Anti tumour necrosis-alpha therapy increases the number of FOXP3 regulatory $\mathrm{T}$ cells in children affected by Crohn's disease. Immunology. 2008;2:178-183.

15. Vos AC, Wildenberg ME, Duijvestein M et al. Anti-tumor necrosis factor- $\alpha$ antibodies induce regulatory macrophages in an Fc region-dependent manner. Gastroenterology. 2011;1:221-230.

Submitted: 2 July, 2013 Accepted after reviews: 16 September, 2013 